\title{
LE TUBERCULOME INTRA MEDULLAIRE : UNE CAUSE RARE DE PARAPARESIE
}

\section{INTRA MEDULLARY TUBERCULOMA : A RARE CAUSE OF PARAPARESIS}

HAIDARA Aderehime 1

VARLET Guy ${ }^{1}$

N'DRI OKA Dominique ${ }^{1}$

BROALET Maman You Epérance ${ }^{1}$

DOUKOURE Brahima ${ }^{2}$

N'DA Herman ${ }^{1}$

DROGBA Kporou Landry ${ }^{1}$

1. Service de neurochirurgie du CHU d'Abidjan Yopougon

2. Service d'anatomie pathologique, CHU de Cocody, Abidjan, Cote d'Ivoire

E-Mail Contact - HAIDARA Aderehime : ader_medic (at) yahoo (dot) fr

Mots clés : tuberculome, lésion intra médullaire, chirurgie

keys words : intramedullary lesion - spinal cord - tuberculoma

\section{RESUME}

Le tuberculome intra-médullaire (TIM) est une localisation de la tuberculose du système nerveux central. Nous rapportons un cas de TIM, chez un patient de 48 ans, sans antécédents particuliers, qui a consulté pour un déficit moteur des 2 membres inférieurs d'installation progressive. L'examen clinque a permis d'objectiver un syndrome de compression médullaire thoracique. La découverte d'une masse intra médullaire, après les explorations neuroradiologiques (myéloscanner et IRM), nous a fait poser l'indication d'une exérèse micro-chirurgicale. Le diagnostique de TIM a été affirmé par l'examen anatomo-pathologique de la pièce opératoire. L'association d'une chimiothérapie anti-tuberculeuse au delà de 6 mois après l'exérèse chirurgicale, à permis une guérison complète après un recul de 18 mois.

\section{SUMMARY}

The intra-medullary tuberculoma (IMT) is a rare tuberculosis located in the central nervous system. We report a case of IMT in a 48-year-old man with a progressive paresis of the lower limbs. The patient had previously been healthy. The neuroradiologic investigations showed an intra-medullary mass. A total exeresis was performed via a micro-surgical approach. The IMT diagnosis was confirmed by the anatomopathological examination.The association of an anti-tuberculosis chemotherapy for 6 months after the surgery led to a complete healing after an 18 months lapse. 


\section{INTRODUCTION}

La tuberculose constitue un véritable problème de santé publique dans le monde, mais surtout dans les pays d'Afrique et d'Asie $[2,5,7,8,10,11,15,17,18]$. En outre, la pandémie du SIDA, a favorisé au cours de ces dernières décennies, une recrudescence des cas de tuberculose. La localisation au niveau du système nerveux (SNC) est rare ; Elle représente environ 0,5 à $2 \%$ des patients tuberculeux $[5,8,14,15,16]$. Le tuberculome du SNC est encore plus rare, il se développe le plus souvent dans le parenchyme cérébral. La location intra-médullaire du tuberculome représente $0.02 \%$ des atteintes tuberculeuses du SNC $[1,2,5,6,8,9,14,15,16]$.

Le tuberculome intra-médullaire (TIM) a été très peu rapporté dans Littérature [9]. II se manifeste habituellement par un tableau de compression médullaire lente, et les progrès L'IRM permettent aujourd'hui de mieux préciser ses caractéristiques radiologiques. Nous rapportons le seul cas observé dans le service de Neurochirurgie d'Abidjan. Le diagnostic de TIM a été fait par l'examen anatomo-pathologique de la pièce opératoire après la chirurgie. Les aspects épidémiologiques, cliniques, radiologiques et les modalités thérapeutiques sont discutés à l'aide de la littérature.

\section{OBSERVATION}

Monsieur O.A, âgé de 48 ans, à consulté pour des troubles de la marche à type de fatigabilité, d'évolution aggravante depuis 2 mois. II se plaignait des paresthésies, de dorsalgies et de troubles vésico-sphinctériens d'apparition récents à type de dysurie. La symptomatologie évoluait dans un contexte de subfébrile, avec un amaigrissement progressif. Ses antécédents médicaux étaient sans particularités. II n'y avait pas de notion de contage tuberculeux. L'examen clinique retrouvait un état général relativement bien conservé, Une para parésie spastique cotée à $4 / 5$ droite et $3 / 5$ à gauche, une hypo-esthésie à niveau T12. Les reflexes rotuliens et achilléens étaient vifs et il existait un signe de Babinski bilatéral. Le bilan biologique montrait une anémie normochrome normocytaire, une vitesse de sédimentation légèrement accélérée, Le bilan rénal était sans particularité, et la sérologie HIV était négative. Un myéloscanner thoracique initialement réalisé montrait un élargissement du cône médullaire évoquant la présence d'une tumeur intramédullaire (figure 1). L'IRM visualisait la lésion sous forme de signal hétérogène avec une composante périphérique se rehaussant après injection du produit de contraste et centrée par une hypo-intense (figure 2). On retrouvait également un signal hypo intense sur les différentes séquences, à la périphérie de la lésion, témoignant de l'existence d'un œdème péri-lésionnel. Le diagnostique d'astrocytome intra médullaire était évoqué et l'indication de l'exérèse microchirurgicale était retenu. Une corticothérapie a été instituée avant la chirurgie. Une laminectomie de T12-T11 a été réalisée. II n'y avait aucune anomalie extradurale en dehors d'une expansion du cône médullaire témoignant de la présence d'une masse intradurale. Sous microscope opératoire, une myélotomie longitudinale permettait l'exérèse en monobloc, d'une masse ovalaire mesurant $3 X 2 X 1 \mathrm{~cm}$, d'aspect jaunâtre, de consistance ferme et granuleuse. Cette lésion se décollait facilement du tissu nerveux. L'examen anatomopathologique de la pièce opératoire mettait en évidence une lésion folliculaire centrée par une nécrose caséeuse plus ou moins complète entourée de cellules épithélioïdes, de cellules géantes de Langhans et des lymphocytes en périphérie. La coloration de Ziehl-Nielsen n'a pas permis d'isoler des Bacilles Acido-Alcoolo-Résistants (BAAR). Le diagnostic de TIM a alors été retenu (Figure 3). L'évolution post-opératoire a permis de constater à $\mathrm{J} 3$ une récupération partielle du déficit moteur et des troubles vésicosphinctériens. Le patient a été mis sous traitement anti-tuberculeux pendant 10 mois et des séances de rééducation fonctionnelle ont été prescrites. L'évolution à 18 mois était satisfaisante avec une reprise autonome de la marche .L'IRM de contrôle n'a montré pas de récidive de la lésion tuberculeuse.

\section{DISCUSSION}

L'atteinte médullaire de la tuberculose est le plus souvent secondairement à une localisation vertébrale ; c'est la classique spondylodiscite à BK ou mal de POTT. Par ailleurs, le Mycobactérium tuberculeux peut atteindre directement le tissu nerveux. Les lésions tuberculeuses vont se développer soit dans l'espace épidural ou dans l'espace sous-dural par diffusion à travers les méninges, soit dans le parenchyme cérébral ou médullaire. II se produit une réaction inflammatoire locale qui va évoluer vers la formation du granulome. [ 7,13]. La localisation du tuberculome dans le parenchyme cérébral est plus fréquente que dans le tissu médullaire, avec un ratio de $1 / 42[2,3,4,8,15,17,18]$. Le rapport de poids, et la différence de vascularisation, plus importante au niveau cérébral, pourraient expliquer cette disproportion [3,9,17]. L'atteinte médullaire se fait généralement par voie hématogène à partir d'un foyer tuberculeux développé à distance dans 
l'organisme. Habituellement, il s'agit d'une tuberculose pulmonaire évolutive $[4,5,7,13,18]$. Dans le cas présent, l'atteinte médullaire était isolée. Le TIM survient le plus souvent chez le sujet jeune entre 20 et 30 ans $[3,16,17]$, sa fréquence plus élevée chez des sujets atteints de $\mathrm{VIH}$, ou faisant l'objet d'un traitement immunosuppresseur $[1,7,9,11,15,18]$. la sérologie HIV de notre patient était négative. Une telle séronégativité a également été retrouvée chez différents patients dans d'autres études $[4,13,14,16$, ]. Le segment médullaire thoracique est le siège plus fréquent du TIM. Les autres segments cervical et lombaire sont plus rarement concernés $[1,6,9]$.

Le tableau clinique de la TIM est semblable à celui d'autres tumeurs intra-médullaires [17]. II s'agit généralement d'un syndrome de compression médullaire lente dont l'expression clinique est fonction du siège de lésion $[5,7,9,15,16]$. La survenue d'un déficit moteur d'aggravation rapide a été rapportée par certains auteurs $[9,18]$. L'association de signes d'imprégnations tuberculeuses tels que la fièvre, les sueurs nocturnes, et la notion de contage tubage tuberculeux peuvent être retrouvés à l'interrogatoire [5]. Dans notre cas, il $s$ agissait d'un déficit moteur d'installation lente dans un contexte d'amaigrissement et de fièvre vespérale. La myélographie et le myéloscanner permettent de mettre en évidence une image de grosse moelle avec la présence d'une lésion intra-médullaire $[6,15,18]$, mais ces examens sont aujourd'hui de moins en moins réalisés. L'IRM précise mieux les caractéristiques radiologiques de la TIM, permettant une approche diagnostique plus aisée [2,3,4,5,7,8,9,14,16]. Rhoton [12] a été le premier à décrire ces caractéristiques en 1988. actuellement, avec les progrès de l'IRM, deux aspects du TIM sont décrits en fonction du stade évolutif $[3,5,6,15,16,17]$. Au stade initial, il existe une réaction inflammatoire avec un œdème périphérique plus ou important, la capsule est pauvre en collagène, et le tuberculome apparaît isointense aussi bien en séquence $\mathrm{T} 1$ et $\mathrm{T} 2$ avec un rehaussement homogène avec après injection. $A$ un stade plus tardif, la capsule du tuberculome s'enrichit en collagène et la réaction inflammatoire circulaire décroit en intensité ou disparaît. La lésion apparaît hypo intense en séquence t1 et iso a hypo intense en T2 avec une prise de contraste annulaire centre par une image hypo intense. Le centre de la lésion devient hyper intense en T2 avec l'apparition du caséum. La partie périphérique du granulome peut apparaître hypo à hyper intense en T2 en fonction du stade d'évolution. L'œdème péri lésionnel se présente comme une image hyper intense en T2. Dans notre cas il s'agissait d'une lésion mixte fusiforme, se rehaussant en T1 après injection de gadolinium avec une partie centrale hypo intense. Plusieurs auteurs rapportent l'existence de tuberculomes multiples à l'IRM, de même que la présence simultanée de tuberculome intra-cérébral $[9,10,14,18]$. YEN [18] recommande la réalisation systématique d'une IRM cérébrale chez des personnes présentant des TIM multiples à cause du caractère très souvent asymptomatique des ces localisations.

Le consensus sur le traitement de la TIM n'est pas encore clairement établi $[9,13]$. L'utilisation de corticoïdes est controversée, car ils n'ont pas fait la preuve de leur efficacité. [5,6]. Cependant leur action sur l'œdème péri lésionnel, et l'amélioration des troubles neurologiques observée chez certains patients justifient leur prescription [4,13]. Le traitement anti-tuberculeux (TAT) doit être le traitement de première intention $[3,4,5,7,14]$. Lorsque le diagnostic de TIM est retenu, l'administration précoce du traitement antituberculeux, au delà de 6 mois, permet le plus souvent une guérison complète $[8,16]$. Les indications de la chirurgie ne sont pas clairement définies dans la littérature [7]. Quoi qu'il en soit, il doit être réservée à certaines conditions notamment, en cas de doute diagnostic, une augmentation de la taille du tuberculome ou l'aggravation du déficit neurologique malgré un traitement médical bien conduit [2, 3,5]. Toutefois en pratique, la découverte d'une masse intra-médullaire bien circonscrite, associée à la présence de troubles neurologique, motivent le plus souvent l'exérèse chirurgicale de la lésion sans délai comme ce fut cas chez notre patient. Le diagnostique de TIM est affirmé à posteriori par l'examen anatomo-pathologique de la pièce opératoire et le traitement anti-tuberculeux est immédiatement institué $[4,9,17]$.

\section{CONCLUSION}

Le TIM, bien qu'étant une affection rare doit être évoqué devant un tableau de compression médullaire lente, surtout dans notre contexte. L'apport des moyens neuroradiologiques modernes dans son diagnostique, l'utilisation des techniques microchirurgicales et l'association d'un traitement anti-tuberculeux adéquat le rendent aujourd'hui potentiellement curable. 


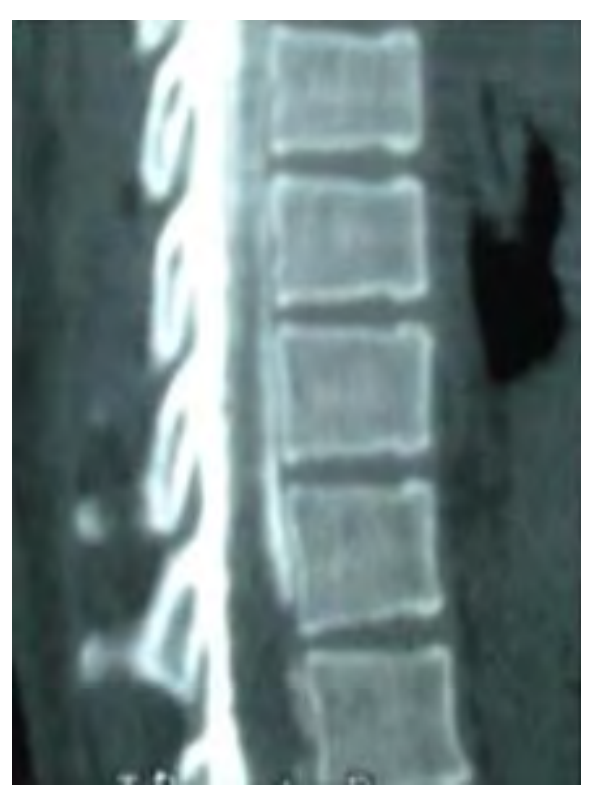

Figure 1

Myeloscanner reconstruction sagittale montrant un élargissement du cône médullaire

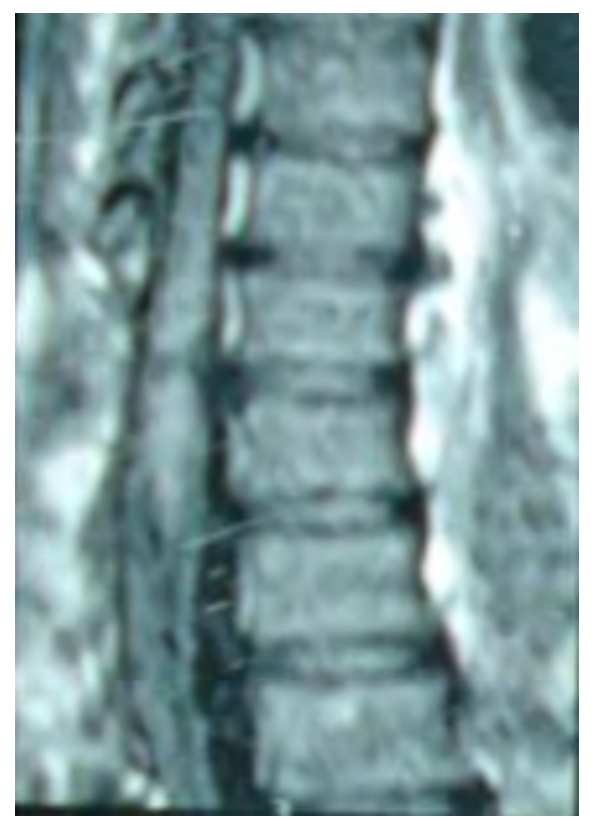

Figure 2

IRM médullaire : lésion mixte avec une composante périphérique se rehaussant après injection de Gadolinium, centré par une image hypo intense 


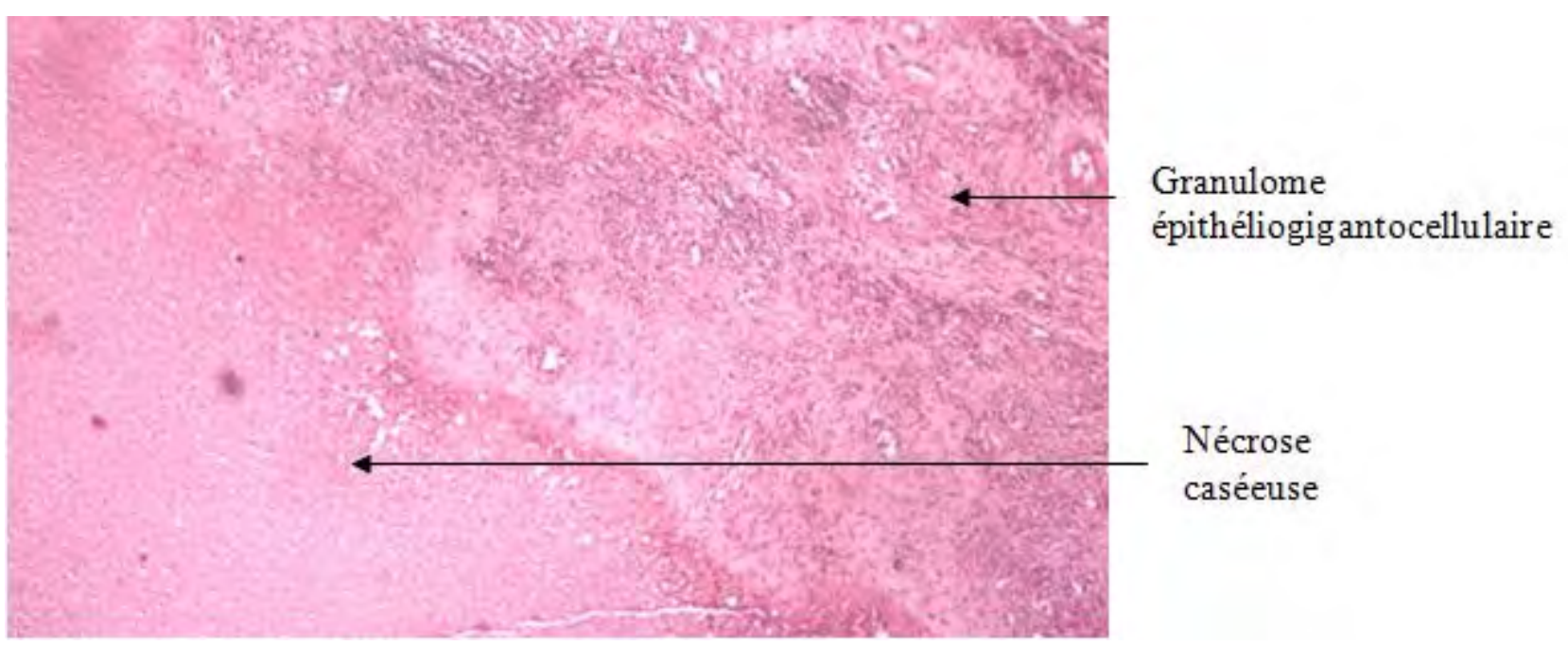

Figure 3

HEX40 : Tuberculose caséo-folliculaire : plage de nécrose caséeuse entourée d'un granulome épithéliogiganto-cellulaire. 


\section{REFERENCES}

1. ARSLANTAS A, FARUK A, KISMET B, ESREF T. Intra-medullary tuberculoma of the spinal cord. J post grad Med 2002; 48:54-5.

2. CITOW JS, AMMIRATI M. Intramedullary tuberculoma of the spinal cord. Neurosurgery 1994; 35:327-30.

3. DEVI BI, CHANDRA S, MONGIA S, CHANDRAMOULI BA, SASTRY KV, SHANKAR SK. Spinal intramedullary tuberculoma and abscess: a rare cause of paraparesis. Neurol India 2002; 50:494-6.

4. HODA MF, PRASAD R, SINGH V.P, MAURYA P. Spinal intramedullary tubercular abcess. Indian J Tuberc 2005; 52:211-214.

5. JAISWAL AK, JAISWAL S, GUPTA SK,SINGH GAUTAM VK, KUMAR S. Intramedullary tuberculoma of the conus. J Clin Neurosci 2006;13:870-2.

6. MIYAMOTO J, SASAJIMA H, OWADA K, ODAKE G, MINEURA K. Spinal Intramedullary Tuberculoma Requiring Surgical Treatment. Neurol med-chir 2003; 43:567-571.

7. KAYAOGLU CR, TUZUN Y, BOGA Z, ERDOGAN F, GORGUNER M, AYDIN IH. Intramedullary spinal tuberculoma.Spine 2000; 25:2265-8.

8. KEMALOGLU S, GUR A, NAS K, CEVIK R, BUYUKBAYRAM H, SARAC AJ. Intramedullary tuberculoma of the conus medullaris. Spinal Cord 2001; 39:498-501.

9. MUTHUKUMAR N, VENKATESH G, SENTHILBABU S, RAJBASKAR R. Surgery for intramedullary tuberculoma of the spinal cord. Surgical Neurology 2006; 66:69-74.

10.PIKE J, ,STEINBOK P, REILLY CW. Cervical intramedullary tuberculoma and tuberculous kyphosis in a 23-month-old child. Can J Surg $2005 ;$ 48:247-50.

11.RATLIFF JK, CONNOLLY ES. Intramedullary tuberculoma of the spinal cord. Case report and review of the literature. J Neurosurg 1999; 90:125-8.

12.RHOTON EL, BALLINGER WE JR, QUISLING R, SYPERT GW. Intramedullary spinal tuberculoma. Neurosurgery 1988 Apr; 22:733-6.

13.SHAHARAO VB, PAWAR M, AGARWAL R, BAVDEKAR SB. Intra-medullary tuberculoma occurring during treatment of tuberculous meningitis. Indian J Pediatr 2004; 71:107-8.

14.SHENOY SN, RAJA A. Concurrent intramedullary and intracerebral tuberculomas. Neurol India 2004; 52:514-6.

15.SUZER T, COSKUN E, TAHTA K, BAYRAMOGLU H, DUZCAN E. Intramedullary spinal tuberculoma presenting as a conus tumor. Eur Spine J 1998; 7:168-71.

16.TORII H, TAKAHASHI T, SHIMIZU H, WATANABE M, TOMINAGA T. Intramedullary spinal tuberculoma. Neurol Med Chir 2004; 44:266-8.

17.Tureyen K. Tuberculoma of the conus medullaris. Neurosurgery 2002; 50:651-2.

18. YEN HL, LEE RJ, LIN JW, CHEN HJ. Multiple tuberculomas in the brain and spinal cord. Spine 2003; 28:499-502. 